\title{
A Generalization of the Hopf-Cole Transformation ${ }^{\star}$
}

\author{
Paulius MIŠKINIS
}

Department of Physics, Faculty of Fundamental Sciences, Vilnius Gediminas Technical University, Sauletekio Ave 11, LT-10223, Vilnius-40, Lithuania

E-mail: paulius.miskinis@vgtu.lt

Received June 04, 2012, in final form February 17, 2013; Published online February 25, 2013

http://dx.doi.org/10.3842/SIGMA.2013.016

\begin{abstract}
A generalization of the Hopf-Cole transformation and its relation to the Burgers equation of integer order and the diffusion equation with quadratic nonlinearity are discussed. The explicit form of a particular analytical solution is presented. The existence of the travelling wave solution and the interaction of nonlocal perturbation are considered. The nonlocal generalizations of the one-dimensional diffusion equation with quadratic nonlinearity and of the Burgers equation are analyzed.
\end{abstract}

Key words: nonlocality; nonlinearity; diffusion equation; Burgers equation

2010 Mathematics Subject Classification: 26A33; 35K55; 45K05

\section{Introduction}

The classical Hopf-Cole transformation is applied for the solution of the nonlinear diffusion equation. There are two well-known nonlinear generalizations of the diffusion equation: with quadratic nonlinearity and the Burgers equation. The first of them has applications in dealing with plasma and acoustic phenomena [15]. The Burgers equation was initially proposed by $\mathrm{H}$. Bateman while modelling the weak viscous liquid motion [4] and later rediscovered by Burgers as a simple nonlinear partial differential equation in studies on turbulence [8]. This equation can be viewed as a simplified version of the Navier-Stokes equation and related to the heat equation via the Hopf-Cole transformation $[12,20]$. Presently, the number of applications of the Burgers equation is immense (see, for instance, $[17,41]$ and references below).

In the case when the properties of a system in a certain point of configuration or phase space depend not only on the properties of this system at this point, but also on the properties of at least one point of the environment, we deal with the nonlocal phenomena. As the examples, let us mention the well-known prey-predator system of Volterra with delay in ecology [40], the ferromagnetic properties of matter in physics [5], viscoelastic phenomena in mechanics [26]. From the mathematical point of view, such phenomena are usually described by the integrodifferential equations [2]. Over the last few years, more attention has been given to a special part of the theory of integro-differential equations, the so-called fractional calculus [32,33,37]. This approach is applied not only in the theory of fractals and to the above-mentioned, already classical, nonlocal phenomena, but also for the description of electrical, biological and diffusion phenomena. The latter topic, as follows from the growing number of publications, receives much attention [33].

Some time ago, two fractional generalizations of the classical diffusion equation were proposed. One of them leads to replacing the second space derivative by the fractional one

$$
\phi_{t}-\alpha_{a} D_{x}^{2+p} \phi=0
$$

${ }^{\star}$ This paper is a contribution to the Special Issue "Geometrical Methods in Mathematical Physics". The full collection is available at http://www.emis.de/journals/SIGMA/GMMP2012.html 
where $\phi=\phi(x, t),{ }_{a} D_{x}^{p}$ is a fractional derivative in the sense of Caputo [33,37], where $0<p<1$, and $a$ is a parameter of nonlocality.

From the physical point of view, we may consider this spatial fractional derivative as a Fourier transformation of the fractional power of the wave number $k$ (see a short review in Appendix B).

From this approach, but for the wave equation, the fractional derivative was considered by A.N. Gerasimov in [16]. The other generalization, proposed by R.R. Nigmatullin in [30], is related to the fractional substitution of the time derivative

$$
{ }_{a} D_{t}^{p} \phi-\alpha \phi_{x x}=0 .
$$

In this situation, due to the well-known relation between the diffusion and the Burgers equations, we may expect two analogous nonlocal generalizations of the Burgers equation

$$
\phi_{t}+\phi \phi_{x}-\alpha_{a} D_{x}^{p} \phi=0
$$

and the other one

$$
{ }_{a} D_{t}^{p} \phi+\phi \phi_{x}-\alpha \phi_{x x}=0 .
$$

These generalizations (1.2) and (1.3) are just the applications of the ideas of [16,30]. Usually, equation (1.3) is called the fractional Burgers equation. However, most interesting and perhaps most productive is the third, fractional Burgers equation with nonlocal nonlinearity (FBENN), i.e. the nonlinear and nonlocal generalization of the diffusion equation, based on a fractional generalization of the Hopf and Cole transformation

$$
\phi_{t}+\frac{1}{2}{ }_{a} D_{x}^{p}\left({ }_{a} D_{x}^{1-p} \phi\right)^{2}-\alpha \phi_{x x}=0,
$$

in which $\phi(x, t), \phi_{0}(x) \in \mathbb{R},-\infty<x<+\infty ; t \geq 0$ and the parameter $\alpha>0$. In this equation, ${ }_{a} D_{x}^{p} \phi=\lambda^{p-1}{ }_{a} \partial_{x}^{p} \phi$ is the fractional derivative, where $\lambda$ is the length parameter, and ${ }_{a} \partial_{x}^{p} \phi$ is the Caputo fractional derivative (see Appendix B). Thus, all the terms of the FBENN have integer physical dimensions. From the mathematical point of view, in the case of linear equation, the transition to the dimensionless form is recommended, and in the case of nonlinear equation such transition is necessary (for details, see Appendix A).

A series of exact analytical solutions of this equation, the asymptotic form of the solutions and a fractional generalization of the Reynolds number are presented. Concrete examples corresponding to the simplest behavior of fractal solution are analyzed.

\section{Linearization}

The main problem in solving the FBENN (1.4) is its nonlinearity. If for the linear integrodifferential equation we may apply some powerful methods [34,36], in the nonlinear case we can get only general estimates [24]. Therefore, the application of the old method of linearization could be helpful in understanding some properties of the FBENN.

Let $\bar{\phi}$ be a known solution of the FBENN (1.4), and $\phi=\bar{\phi}+\varepsilon \psi$ is a small perturbation of solution $\bar{\phi}$, where $\psi=\psi(x, t)$. Then $\psi(x, t)$, in the first order of $\varepsilon$, obeys the linear equation

$$
\psi_{t}+\bar{\phi}_{x a} D_{x}^{1-p} \psi-\alpha \psi_{x x}=0 .
$$

The solution $\bar{\phi}$ could be obtained by any method presented in the next sections. Thus, according to the general theorems of the uniqueness and existence of the weak sense solution given in equation (6.3) below, the solution of the linearized FBENN (2.1) could be found numerically.

The asymptotic behavior of the linearized solution $\psi(x, t)$ at $t \rightarrow \pm \infty$ is very important for the stability of solution $\phi(x, t)$. 


\section{Nonlocal perturbations of the local solutions}

Let us take advantage of the fact that in the case of $p=0$ and $p=1$ the solutions of the respective equations are known. To find how the nonlocality changes of the local solution of the FBENN (1.4), we shall perform the expansion of the latter two equations in the neighbourhood of $0+\varepsilon$ and $1-\varepsilon$ of the parameter $p$.

In the neighbourhood of the point $p=0+\varepsilon$, the fractional derivative has the expansion

$$
{ }_{a} D_{x}^{p} \psi(x)=\phi(x)+\varepsilon_{a} \hat{N}_{x} \phi(x)+O\left(p^{2}\right),
$$

here ${ }_{a} \hat{N}_{x} \phi(x)$ is the nonlocal operator

$$
{ }_{a} \hat{N}_{x} \phi(x)=-\gamma \phi(x)-\int_{a}^{x} \phi^{\prime}(\xi) \log (x-\xi) d \xi
$$

here $\gamma$ is the Euler constant.

Then the expansion of the FBENN (1.4) in the neighbourhood of the respective points is

$$
\begin{aligned}
\phi_{t}+\frac{1}{2} \phi_{x}^{2}-\alpha \phi_{x x} & =\varepsilon\left[\phi_{x}\left({ }_{a} \hat{N}_{x} \phi\right)_{x}-\frac{1}{2}{ }_{a} \hat{N}_{x} \phi_{x}^{2}\right], & & p=0+\varepsilon \\
\phi_{t}+\phi \phi_{x}-\alpha \phi_{x x} & =(1-\varepsilon)\left[{ }_{a} \hat{N}_{x} \phi^{2}-\phi_{a} \hat{N}_{x} \phi\right]_{x}, & p & =1-\varepsilon .
\end{aligned}
$$

Thus, as follows from equations (3.1) and (3.2), in the case of weak nonlocality (small values of the parameters $p$ or $1-p$ ) FBENN (1.4) can be interpreted as the respective classical equations perturbed by nonlocal terms of a highly specific form. Both these equations can be solved numerically.

\section{The travelling wave solution}

Let us change the reference frame and turn to a new variable $\xi=x-u t$. Then the FBENN (1.4) takes the form

$$
\frac{1}{2}{ }_{a} D_{\xi}^{p}\left({ }_{a} D_{\xi}^{1-p} \phi\right)^{2}=\alpha \phi^{\prime \prime}+u \phi^{\prime}
$$

The solutions of the corresponding equations for $p \rightarrow 0$ and $p \rightarrow 1$ are known

$$
\begin{aligned}
\phi(\xi) & =2 u \xi-2 \alpha \ln \left[e^{\frac{u \xi}{\alpha}+2 u C_{1}}-1\right]+C_{2}, \\
\phi(\xi) & =u\left(1-\operatorname{th}\left[\frac{u}{2 \alpha}(\xi+C)\right]\right) .
\end{aligned}
$$

Suppose that according to the "intermediate" character of the evolution equation (4.1), the travelling wave solution $\phi(\xi)$ of this equation is just a fractional "deformation" of the solution $\phi^{(0)}(\xi)\left(\phi^{(1)}(\xi)\right)$ with the integer value of the parameter $p=0(p=1)$

$$
\phi(\xi)={ }_{a} D_{\xi}^{p} \phi^{(0)}(\xi), \quad \phi(\xi)={ }_{a} D_{\xi}^{p-1} \phi^{(1)}(\xi),
$$

where ${ }_{a} D_{\xi}^{p-1} f(\xi) \equiv{ }_{a} I_{\xi}^{1-p} f(\xi)$ means a fractional integral (see Appendix B). Indeed, the substitution of expressions (4.3) into travelling wave equation (4.1) leads to equations for the corresponding solutions $\phi^{(0)}(\xi)$ and $\phi^{(1)}(\xi)$.

As a consequence, from $(4.3)$ we get $\phi^{(1)}(\xi)=\partial_{\xi} \phi^{(0)}(\xi)$. The assumption (4.3) allows us to get the travelling wave solution of the equation (4.1) by substituting the corresponding solutions of this equation for the limit cases $p \rightarrow 0$ and $p \rightarrow 1$ into expression (4.3). 
The travelling wave solution of (4.1) is

$$
\phi=2 u \xi-2 \alpha \log \left[\exp \frac{u(c+\xi)}{\alpha}-1\right], \quad p=0,
$$

with the asymptotics $\phi=-2 u c, \xi \gg 1$;

$$
\phi=-\frac{2}{\Gamma(2-p)} \frac{u \xi+\frac{p}{\alpha} u^{2}-(1-p) C}{(\xi-a)^{p}}, \quad 0<p<1,
$$

with the integration constant $C$, and

$$
\phi=\phi_{1}+\frac{\phi_{2}-\phi_{1}}{1+\exp \left(\frac{\phi_{2}-\phi_{1}}{2 \alpha} \xi\right)}, \quad p=1,
$$

with the asymptotics $\phi(\xi \rightarrow+\infty)=\phi_{1}, \phi(\xi \rightarrow-\infty)=\phi_{2}, \phi_{2}>\phi_{1}$.

\section{The fractional generalization of the Hopf-Cole transformation}

In the case of the evolutional Burgers equation (BE),

$$
\phi_{t}+\phi \phi_{x}-\alpha \phi_{x x}=0
$$

well known is the simple nonlinear Hopf-Cole transformation

$$
\phi(x, t)=-2 \alpha \frac{w_{x}(x, t)}{w(x, t)},
$$

which relates any solution $w(x, t)$ of the diffusion equation $(\mathrm{DE}) w_{t}=\alpha w_{x x}$ to the solution $\phi(x, t)$ of the BE.

In the case of the nonlinear diffusion equation (NDE) with quadratic nonlinearity

$$
\phi_{t}+\frac{1}{2} \phi_{x}^{2}-\alpha \phi_{x x}=0
$$

there is also a nonlinear transformation, which relates the solution $w(x, t)$ to the solution $\phi(x, t)$ of the NDE (5.3)

$$
\phi(x, t)=-2 \alpha \log w(x, t) .
$$

We see that the above two transformations are the cases of one and the same transformation which is as follows

$$
\phi(x, t)=-2 \alpha_{a} D_{x}^{p} \log w(x, t),
$$

for $p=0$ (5.4) and $p=1$ (5.2), and the transformation (5.5) itself relates the diffusion equation solution $w(x, t)$ to the FBENN (1.4) solution $\phi(x, t)$.

It is more expedient, however, particularly in applications, to use the fractional generalization of the Hopf-Cole transformation

$$
\phi(x, t)=-2 \alpha_{a} D_{x}^{p} \log [b+w(x, t)], \quad b \in \mathbb{R} .
$$

Indeed, by substituting expression (5.6) into the initial FBENN (1.4), we obtain the equation

$$
{ }_{a} D_{x}^{p}\left[\frac{w_{t}-\alpha w_{x x}}{b+w}\right]=0
$$

which turns into an identity when $w(x, t)$ is the solution of the DE. 
The point is that by changing the scale and the variables we can always obtain the solution of the diffusion equation for $\|w(x, t)\| \ll 1$. In this case, the generalization of the fractal Hopf-Cole transformation (5.6) becomes even simpler

$$
\phi(x, t)=-2 \alpha_{a} D_{x}^{p} w(x, t) .
$$

From the formula (5.7) in particular it follows that if the diffusion equation solution asymptotically approaches zero, for instance, for $w(x, t) \rightarrow x^{-q}$ for $x \rightarrow+\infty(q>0)$, then the FBENN solution also approaches zero: $w(x, t) \rightarrow x^{-(p+q)}$ for $x \rightarrow+\infty,(q>0)$. In the case of the exponential asymptotic, more convenient is the expression (5.5).

Let $w(x, t): x \in[0,+\infty) \cup t \in[0,+\infty)$. The fractional derivative is the right Caputo derivative in the Weyl sense. In the case of the solution of the diffusion equation

$$
w(x, t)=\exp \left(-\frac{c x}{2 \alpha}+\frac{c^{2} t}{4 \alpha}-b\right)
$$

the solution of the FBENN is

$$
\begin{aligned}
& \phi(x, t)=-2 \alpha \log \left[a+\exp \left(-\frac{c x}{2 \alpha}+\frac{c^{2} t}{4 \alpha}-b\right)\right], \quad p=0, \\
& \phi(x, t)=\frac{c}{a+\exp \left(-\frac{c x}{2 \alpha}+\frac{c^{2} t}{4 \alpha}-b\right)}, \quad p=1, \\
& \phi(x, t)=-2 \alpha_{+\infty} D_{x-}^{p} \log \left[a+\exp \left(-\frac{c x}{2 \alpha}+\frac{c^{2} t}{4 \alpha}-b\right)\right], \quad 0<p<1 .
\end{aligned}
$$

Note that solution (5.10) continuously transforms from solution (5.8) into (5.9) when the parameter $p$ runs from $p=0$ to $p=1$.

It is the fractional generalization of the Hopf-Cole transformation (5.2) that has been used to derive the solution (5.10) which interrelates the diffusion and the FBENN solutions.

Thus, if $T^{(p)}$ is a fractional generalization of the Hopf-Cole transformation, then the interrelation among the nonlinear diffusion equation (NDE), the Burgers equation (BE), the simple diffusion equation (DE) and the nonlinear nonlocal diffusion equation can be graphically shown as follows

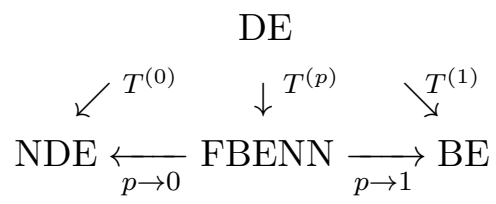

\section{The interrelation of the solutions}

The existence of the fractional generalization of the Hopf-Cole transformation may produce the impression that the properties of the FBENN solutions can be reduced to the nonlocally transform solution of the diffusion equation. Below, we shall show this is not the case.

It is well-known that any solution $w(x, t)$ of the DE $w_{t}=\alpha w_{x x}$ under the simple HopfCole transformation $\phi(x, t)=-2 \alpha w_{x}(x, t) / w(x, t)$ turns into the solution $\phi(x, t)$ of the $\mathrm{BE}$ $\phi_{t}+\phi \phi_{x}-\alpha \phi_{x x}=0$. The reverse is not true, as the solution $\phi(x, t)$ does not obey the DE: if we substitute $\phi(x, t)$ into the $\mathrm{BE}$, we obtain the equation which will be more general than the DE

$$
w_{t}-\alpha w_{x x}=f(t) w,
$$

here $f(t)$ is any time function. 
Thus, there exist the BE solutions that can be expressed through solutions of the simple DE, and the proper solutions that are devoid of such representation.

In a similar way, we can classify also the NDE solutions. Some of them are related to DE solutions through the transformation $\phi(x, t)=-2 \alpha \log w(x, t)$. However, there exist also the proper solutions that are not related to DE solutions.

It is important that the interrelation of the solutions in the case of the travelling wave equation (4.3) can be generalized and is valid for the NDE, FBENN and BE cases

$$
\phi(x, t)={ }_{a} D_{x}^{p} \phi^{(0)}(x, t), \quad \phi(x, t)={ }_{a} D_{x}^{p-1} \phi^{(1)}(x, t),
$$

here, as above, $\phi^{(0)}(x, t)\left(\phi^{(1)}(x, t)\right)$ is the NDE (BE) solution for $p=0(p=1)$. This means that the solutions of all these three equations, irrespectively of the fractional Hopf-Cole transformation, are interrelated. Hence, in particular, it follows that

$$
\phi^{(1)}(x, t)=\partial_{x} \phi^{(0)}(x, t) .
$$

This interrelation is, in a sense, more general as is valid for both the solutions related to the DE solutions and proper solutions. If we substitute the proper solution $\phi^{(1)}(x, t)$ of the BE, we will be able to restore the proper solutions of the NDE.

Let us consider the initial evolution equation (1.4) together with two limit cases at $p \rightarrow 0$ and $p \rightarrow 1$

$$
\begin{aligned}
& \phi_{t}+\frac{1}{2} \phi_{x}^{2}-\alpha \phi_{x x}=0, \\
& \phi_{t}+\phi \phi_{x}-\alpha \phi_{x x}=0 .
\end{aligned}
$$

For $p=0$ we have a diffusion equation with quadratic nonlinearity or just the nonlinear diffusion equation (NDE) (6.1), for $p=1$ - the Burgers equation (BE) (6.2), for $0<p<$ 1 - the integro-differential equation FBENN (1.4) which we may regard as an "intermediate" evolution equation whose solutions turn into the solution of equation (6.1) or (6.2) depending on $\lim _{p \rightarrow 0} \phi^{(p)}(x, t)$ or $\lim _{p \rightarrow 1} \phi^{(p)}(x, t)$.

The solutions to the Cauchy problem (1.4) have to be understood in some weak sense; there are several options reported, e.g., in [6,7]. In the context of the present study, let us just say that under the weak solution (1.4) we mean the solution of the integral equation

$$
\phi(x, t)=e^{\alpha t \partial_{x x}} \phi_{0}-\frac{1}{2} \int_{0}^{t} e^{\alpha(t-\tau) \partial_{x x}} \cdot{ }_{a} D_{x}^{p}\left({ }_{a} D_{x}^{1-p} \phi\right)^{2}(x, t) d \tau
$$

motivated by the classical Duhamel formula.

It is clear now that in the same manner we can also classify the nonlocal solutions of the FBENN. Some of them, through the fractional Hopf-Cole transformation, are related to the

simple DE. However, there are also a number of proper solutions which have no such relation to the BE.

\section{Evolution of the initial conditions}

Let the initial conditions for FBENN (1.4) and for the diffusion equation be related by the expression

$$
w_{0}(x)=e^{-\frac{1}{2 \alpha} a_{x}^{p} \phi_{0}(x)} .
$$


This allows us to express the solution $\phi(x, t)$ of the FBENN through the initial condition $\phi_{0}(x)$ and the general form of the solution of the diffusion equation:

$$
\phi(x, t)=-2 \alpha_{a} D_{x}^{p}\left[\log \left(1+\frac{1}{\sqrt{4 \pi \alpha t}} \int_{-\infty}^{+\infty} e^{-\frac{|x-y|^{2}}{4 \alpha t}-\frac{1}{2 \alpha} a I_{y}^{p} \phi_{0}(y)} d y\right)\right] .
$$

The expression of the solution of the FBENN (1.4) in the general form (7.1) allows us to analyze the time evolution of the nonlocal solution. Indeed, let $w(x, t)$ be the solution of the diffusion equation $w_{t}=\alpha w_{x x}$. Then the solution of (7.1) can be expressed in the form $\phi(x, t)=$ $-2 \alpha_{a} D_{x}^{p} \log (1+w(x, t))$.

As follows from the FBENN (1.4), depending on the values of the parameter $p$ we deal with not one but with an infinite number or an hierarchy of integro-differential equations. One of the most important properties of the FBENN (1.4) is interrelation between nonlinearity and nonlocality: for the fractional value of the parameter $p$ we have the nonlinear-nonlocal and for the integer positive $p$ only a nonlinear generalization of the Burgers equation. In this hierarchy, due to the substitution $\phi(x, t) \rightarrow{ }_{a} D_{x}^{q-p} \phi(x, t)$, the low-order equations turn into the higher-order ones, but in the inverse direction this transformation is multivalued.

\section{Interaction of nonlinear and nonlocal perturbations}

The relation (7.1) between the solutions of the FBENN and diffusion equation allows to consider an interaction of nonlocal and nonlinear perturbations. Two or more perturbations moving with a different velocity can overtake each other or flow together into a new intensive perturbation. The FBENN also describes the interaction process of two or more moving nonlocal perturbations. The principle of superposition is not valid for the nonlinear FBENN, but it is valid for the linear diffusion equation. The fractional Hopf-Cole transformation (7.1) interrelates the solutions of the nonlocal and nonlinear FBENN and of the linear diffusion equation. Thus, if $w_{i}(x, t)$ are the solutions of the diffusion equation, then $\phi(x, t)=-2 \alpha_{a} D_{x}^{p}\left(\log \sum w_{i}\right)$ are the solutions of the FBENN.

For instance, for two solutions of the diffusion equation in the form

$$
w_{i}(x, t)=a_{i} \exp \left(-\frac{c_{i} x}{2 \alpha}+\frac{c_{i}^{2} t}{4 \alpha}-b_{i}\right), \quad i=1,2,
$$

we obtain a nonlocal and nonlinear interaction of these perturbations:

$$
-\frac{\phi(x, t)}{2 \alpha}= \begin{cases}\log \left(w_{1}+w_{2}\right), & p=0, \\ \frac{1}{\Gamma(1-p)} \frac{d}{d x} \int_{a}^{x} \frac{\log \left[w_{1}(\xi, t)+w_{2}(\xi, t)\right]}{(x-\xi)^{p}} d \xi, & 0<p<1, \\ \frac{c_{1} w_{1}+c_{2} w_{2}}{w_{1}+w_{2}}, & p=1 .\end{cases}
$$

\section{The conservation laws}

In the case of the $\mathrm{BE}(6.2)$, for $x \in E^{1}, \forall t>0, \phi( \pm \infty, t)=\phi_{x}( \pm \infty, t)=0$, we have a conservation value, or the time invariant value inv, of

$$
I^{(1)}=\int_{-\infty}^{+\infty} \phi(x, t) d x=\operatorname{inv},
$$

since

$$
\frac{\partial I^{(1)}}{\partial t}=\int_{-\infty}^{+\infty}\left[\alpha \phi_{x}-\frac{1}{2} \phi^{2}\right]_{x} d x=\left.\left(\alpha \phi_{x}-\frac{1}{2} \phi^{2}\right)\right|_{-\infty} ^{+\infty}=0 .
$$


In the applications, this conservation law is called the "mass" conservation law, because if $\phi(x, t)$ can be a one-dimensional density or a gradient of any physical, chemical or biological magnitude. Then, the $I^{(1)}$ corresponds to its conservation.

In the case of the NDE (4.2), if $\forall t>0, \phi_{x}( \pm \infty, t)=\phi_{x x}( \pm \infty, t)=0$, we again deal with the conservation value

$$
I^{(0)}=\phi(+\infty, t)-\phi(-\infty, t)=\operatorname{inv},
$$

since by applying the derivative $\partial_{x}$ to the evolutionary equation (4.2) followed by integration we obtain

$$
\frac{\partial I^{(0)}}{\partial t}=\frac{\partial}{\partial t} \int_{-\infty}^{+\infty} \phi_{x} d x=\left.\left(\alpha \phi_{x x}-\frac{1}{2} \phi_{x}^{2}\right)\right|_{-\infty} ^{+\infty}=0 .
$$

This conservation value shows that the difference in asymptotic values for any time moment remains unchanged. If, for instance, we deal with the evolution of the potentials, the conservation value $I^{(0)}(9.1)$ shows that the difference of potentials for $x \rightarrow \pm \infty$ does not change. In the case of the FBENN (4.3), we again deal with a conservation value if $\forall t>0,{ }_{a} D_{x}^{2-p} \phi( \pm \infty, t)=$ ${ }_{a} D_{x}^{1-p} \phi( \pm \infty, t)=0$

$$
I^{(p)}=\int_{-\infty}^{+\infty}{ }_{a} D_{x}^{1-p} \phi(x, t) d x=\mathrm{inv}
$$

because

$$
\frac{\partial I^{(p)}}{\partial t}=\int_{-\infty}^{+\infty}\left[\alpha_{a} D_{x}^{2-p} \phi-\frac{1}{2}\left({ }_{a} D_{x}^{1-p} \phi\right)^{2}\right]_{x} d x=\left.\left[\alpha_{a} D_{x}^{2-p} \phi-\frac{1}{2}\left({ }_{a} D_{x}^{1-p} \phi\right)^{2}\right]\right|_{-\infty} ^{+\infty}=0 .
$$

Even this simple example highlights two important properties of the nonlocal conservation law (9.2): it interrelates the conservation values of two different dynamical systems, which can be of different mathematical nature (e.g., in our case these values are integral and discrete).

Note that in the "common" case of the nonlocal BE

$$
\phi_{t}+\phi \phi_{x}-\alpha_{a} D_{x}^{2-p} \phi=0
$$

an analogous conservation integral exists at other asymptotic values

$$
\phi( \pm \infty, t)={ }_{a} D_{x}^{1-p} \phi( \pm \infty, t)=0 .
$$

At this point, it is not difficult to characterize the "mass" conservation law of the nonlinear nonlocal evolution equation

$$
\phi_{t}+\frac{1}{2}{ }_{a} D_{x}^{p}\left({ }_{a} D_{x}^{1-p} \phi\right)^{2}-\alpha_{a} D_{x}^{2-q} \phi=0 .
$$

The magnitude $I^{(p, q)}$ is the invariant of the evolution equation (9.3):

$$
I^{(p, q)}=\int_{-\infty}^{+\infty}{ }_{a} D_{x}^{1-p} \phi(x, t) d x=\operatorname{inv}
$$

for ${ }_{a} D_{x}^{1-p} \phi( \pm \infty, t)={ }_{a} D_{x}^{2-(p+q)} \phi( \pm \infty, t)=0$. However, in this case the integrability is sacrificed, and the fractional generalization of the Hopf-Cole transformation does not exist.

The "energy" of travelling excitation

$$
K=\frac{1}{2} \int_{-\infty}^{+\infty}\left({ }_{a} D_{x}^{1-p} \phi\right)^{2} d x
$$


if $\forall t>0,{ }_{a} D_{x}^{2-p} \phi( \pm \infty, t)={ }_{a} D_{x}^{1-p} \phi( \pm \infty, t)=0$, as in the case of the BE, is not unchangeable, but it is constantly decreasing:

$$
\begin{gathered}
\frac{d}{d t} \frac{1}{2} \int_{-\infty}^{+\infty}\left({ }_{a} D_{x}^{1-p} \phi\right)^{2} d x=-\left.\frac{1}{3}\left({ }_{a} D_{x}^{1-p} \phi\right)^{3}\right|_{-\infty} ^{+\infty}+\left.\alpha\left({ }_{a} D_{x}^{1-p} \phi\right)\left({ }_{a} D_{x}^{2-p} \phi\right)\right|_{-\infty} ^{+\infty} \\
-\alpha \int_{-\infty}^{+\infty}\left({ }_{a} D_{x}^{2-p} \phi\right) \cdot\left({ }_{a} D_{x}^{2-p} \phi\right) d x=-\alpha \int_{-\infty}^{+\infty}\left({ }_{a} D_{x}^{2-p} \phi\right)^{2} d x<0 .
\end{gathered}
$$

Like in the case of the nonlocal "mass" conservation law, the "energy" $K$ (9.4) links the energy of the travelling excitation in the case of the $\mathrm{BE}(p=1)$ and the one-dimensional density of energy in the case of the $\operatorname{NDE}(p=0)$.

\section{Symmetries}

The conservation laws are obviously related to the group of the FBENN automorphisms. In the case of the usual BE, there is a large Lee symmetry group of point transformations, which contains the Galilei, dilaton, and projective transformations and is generated by operators

$$
\begin{aligned}
& D=2 t \partial_{t}+x \partial_{x}-\phi \partial_{\phi}, \quad K=t^{2} \partial_{t}+t x \partial_{x}-\left(t u+\frac{1}{2} x\right) \partial_{\phi}, \\
& P_{1}=\partial_{x}, \quad P_{2}=\partial_{t}, \quad B=2 t \partial_{x}-\partial_{\phi} .
\end{aligned}
$$

Symmetries in the discrete BE have been studied in [18].

An important and in the general case uninvestigated problem is to find the nonlocal and nonclassical symmetries of the FBENN. Some general aspects were already presented in [1]. Promising seems an attempt of computer symmetry analysis, as was done for the nonlinear heat equation in [11].

Note here one property that allows us to get new solutions of the FBENN. Let $v(x, t)$ be a known solution of the FBENN (1.4), and $u(x, t)$ is the solution of the linear equation

$$
u_{t}+\left({ }_{a} D_{x}^{1-p} v\right) u_{x}-\alpha u_{x x}=0 .
$$

Then

$$
\phi(x, t)=-2 \alpha_{a} D_{x}^{p} \log u+v
$$

is a new solution of the FBENN (1.4). At an integer value of $p$ we have the local equation (10.1); in particular, for $p=1$ we obtain a new solution of the classical Burgers equation.

Note here that the existence of transformation $T$ expressed by relation (5.6) allows to solve the problem of the FBENN (1.4) symmetry group. If $G_{1}$ is a symmetry group of the diffusion equation, then $G=T G_{1} T^{-1}$ is a symmetry group of the FBENN (1.4).

Here, we shall note an important feature of the FBENN, related to the FBENN symmetry. Let us apply the operator $\partial_{x}$ to the FBENN and take the operator ${ }_{a} D_{x}^{p}$ beyond the brackets

$$
{ }_{a} D_{x}^{p}\left[{ }_{a} D_{x}^{1-p} \phi_{t}+\left({ }_{a} D_{x}^{1-p} \phi\right)\left({ }_{a} D_{x}^{1-p} \phi\right)_{x}-\alpha\left({ }_{a} D_{x}^{1-p} \phi\right)_{x x}\right]=0 .
$$

On substituting the variables while

$$
x \mapsto y \pm A(t+B), \quad{ }_{a} D_{x}^{p} \phi(x, t) \mapsto{ }_{a} D_{y}^{p} \phi(y, t) \pm A,
$$

where $A$ and $B$ are constants, the evolution FBENN will not change its form. This way of generating the new solutions is particularly efficient not in the case of travelling excitations when $\phi=\phi(x-u t)$, but in the general case when $\phi=\phi(x, t)$. 
One of the ways of constructing exact solutions of some of the nonlinear equations consists in finding the corresponding Bäklund transformations. The Bäklund transformations have been found for the majority of the nonlinear equations that are integrated by the inverse scattering method $[14,31]$.

It is possible to show that the generalization of the Hopf-Cole transformation $T^{(p)}$ is a separate case of the Bäklund transformation

$$
w_{x}+\frac{1}{2 \alpha}(b+w)_{a} D_{x}^{1-p} \phi=0, \quad w_{t}+\frac{1}{2}\left[(b+w)_{a} D_{x}^{1-p} \phi\right]_{x}=0 .
$$

On removing $\phi(x, t)$ from the system we obtain the diffusion equation $w_{t}=\alpha w_{x x}$.

To obtain the FBENN from the system (10.2), we shall perform the following procedure. From the diffusion equation for the function $w(x, t)$ and the identity $\left[w_{t} /(b+w)\right]_{x}=\left[w_{x} /(b+w)\right]_{t}$ follows the condition

$$
\left(\frac{w_{x}}{b+w}\right)_{t}=\alpha\left(\frac{w_{x x}}{b+w}\right)_{x}
$$

and from the first equation of the system (10.2) it follows that

$$
\frac{w_{x}}{b+w}=-\frac{1}{2 \alpha}{ }_{a} D_{x}^{1-p} \phi, \quad \text { and } \quad \frac{w_{x x}}{b+w}=-\frac{\left({ }_{a} D_{x}^{1-p} \phi\right)_{x}}{2 \alpha}+\frac{\left({ }_{a} D_{x}^{1-p} \phi\right)^{2}}{4 \alpha^{2}} .
$$

Substitution of the expressions (10.4) in the condition (10.3) gives us the FBENN (1.4)

$$
{ }_{a} D_{x}^{1-p}\left[\phi_{t}+\frac{1}{2}{ }_{a} D_{x}^{p}\left({ }_{a} D_{x}^{1-p} \phi\right)^{2}-\alpha \phi_{x x}\right]=0 .
$$

The first equation of the system (10.2) is a fractional generalization of the Hopf-Cole transformation

$$
\phi(x, t)=-2 \alpha_{a} D_{x}^{p} \log [b+w(x, t)] .
$$

On substituting expression (10.5) into the FBENN we obtain the following equation

$$
{ }_{a} D_{x}^{p}\left[\frac{1}{b+w}\left(w_{t}-\alpha w_{x x}\right)\right]=0
$$

from which it follows that any solution of the diffusion equation can be transformed, through the generalization (10.5) of the fractional Hopf-Cole transformation, into an FBENN solution. The reverse implication would be incorrect, as from equation (10.6) follows a more general diffusion equation. For instance,

$$
w_{t}-\alpha w_{x x}=\left(C_{1}+f(t)\right)(b+w) .
$$

It is because of this above-mentioned ambiguity of the fractional generalization of the HopfCole transformation that the FBENN solutions are differentiated into those related to the diffusion equation and proper ones.

\section{The asymptotic form of solutions}

A very convenient dimensionless quantity which is used in the nonlinear BE is the Reynolds number. This number is just a ratio of the nonlinear and the dissipative terms: $\operatorname{Re} \sim \phi \phi_{x} / \alpha \phi_{x x}$. In the case when $\operatorname{Re} \ll 1$, the influence of the nonlinear term is negligible, but for $\operatorname{Re} \gg 1$ 
this term plays a crucial role and leads to the nonlinear Riemann equation which describes the simplest type of the shock waves ${ }^{1}$.

For the FBENN (1.4) we may introduce a dimensionless generalization of the Reynolds number, which at the $p=1$ coincides with the classical Reynolds number for the BE

$$
\operatorname{Re} \sim \frac{{ }_{a} D_{x}^{p}\left({ }_{a} D_{x}^{1-p} \phi\right)^{2}}{\alpha \phi_{x x}} \sim \frac{\phi x^{p}}{\alpha \lambda^{1+p}}
$$

where $\lambda$ is the characteristic parameter of length in the model.

Depending on the value of this number, we obtain two limit cases of the FBENN

$$
\begin{aligned}
& \phi_{t}-\alpha \phi_{x x}=0, \quad \operatorname{Re} \ll 1, \\
& \phi_{t}+\frac{1}{2}{ }_{a} D_{x}^{p}\left({ }_{a} D_{x}^{1-p} \phi\right)^{2}=0, \quad \operatorname{Re} \gg 1 .
\end{aligned}
$$

The equation (11.1) is just a diffusion equation, and the equation (11.2) we will call the fractional Riemann equation. In the case of $\operatorname{Re} \gg 1$, depending on the value of the order of the fractional derivative $p$, we have three evolution equations

$$
\begin{aligned}
& \phi_{t}+\frac{1}{2} \phi_{x}^{2}=0, \quad p=0, \\
& \phi_{t}+\frac{1}{2}{ }_{a} D_{x}^{p}\left({ }_{a} D_{x}^{1-p} \phi\right)^{2}=0, \quad 0<p<1, \\
& \phi_{t}+\phi \phi_{x}=0, \quad p=1 .
\end{aligned}
$$

The "mass" conservation law predetermines the asymptotic form of the FBENN solution. To confirm such a result, let us consider some estimates. From the general form of the FBENN solution (7.1) it follows that the limit $t \rightarrow+\infty$ corresponds to a rather low value of the parameter $\alpha$. At a low $\alpha$, to calculate the values of the corresponding integrals we can apply the saddle point approximation.

The critical point $y_{0}$ can be determined from the equation $\frac{y_{0}-x}{t}+{ }_{a} D_{y}^{1-p} \phi_{0}\left(y_{0}\right)=0$. Then the asymptotic expression of the FBENN solution acquires the form

$$
\phi(x, t)={ }_{a} D_{x}^{p}\left[\frac{\left(x-y_{0}\right)^{2}}{2 t}+C\right] \sim \frac{(x-a)^{2-p}}{t \Gamma(3-p)}+C_{1}(x-a)^{-p} .
$$

For $x \rightarrow+\infty$ and $0<p<1$, the solution $\phi(x, t) \rightarrow(x-a)^{2-p} /[t \Gamma(3-p)]$. Thus, we obtain a power-deformed perturbation of the usual solution of the Burgers equation. Note here that these estimates are valid not only in the environment of the meaning $p=1$, but also for any $p \in \mathbb{R}$.

We have to show the region of the validity of solution (11.3). In the limit case, the integral in the expression of the mass conservation law diverges. Therefore, for $x>x_{0}$ the solution $\phi(x) \equiv 0$. To determine the value $x_{0}$, we insert the asymptotic form of solution (11.3) in the expression of the mass conservation law (9.2). This means that $x_{0}^{2} / 2 t \sim I$. Thus, the maximum meaning of the solution

$$
\phi_{\max }(x, t) \sim \frac{I^{1-\frac{p}{2}}}{(2 t)^{\frac{p}{2}}} \quad \text { and } \quad x_{0} \sim \sqrt{2 I t} .
$$

\footnotetext{
${ }^{1}$ This equation has some names: Hopf, weak shock waves, Riemann-Hopf. We are following reference [35].
} 


\section{The supersymmetric nonlinear nonlocal diffusion evolution equation}

We shall show that the FBENN has a supersymmtric generalization. Let the superfield $\chi=$ $\theta_{a} D_{x}^{1-p} \phi+\psi$ unite two fields of different properties: the "bosonic" field $\phi(x, t)$ and its spinor superpartner $\psi(x, t) ; \theta$ is the constant Majorana spinor. The transformations of the fields $\phi, \psi$ are nonlocal because of the fractional derivatives ${ }_{a} D_{x}^{p} f(x)$

$$
\delta_{\eta} \psi=\eta_{a} D_{x}^{1-p} \phi, \quad \delta_{\eta a} D_{x}^{1-p} \phi=\eta \psi_{x} .
$$

However, the commutator of the two transformations (12.1) is a spatial translation

$$
\left[\delta_{\eta}, \delta_{\xi}\right]=2 \xi \eta \partial_{x}
$$

The supersymmtric equation

$$
\chi_{t}=\left(\chi_{x}+\frac{1}{2} \chi \mathcal{D} \chi\right)_{x}
$$

(here $\mathcal{D}=\theta \partial_{x}+\partial_{\theta}$ is a supersymmetric derivative) is a system of two evolutionary equations

$$
\psi_{t}=\psi_{x x}+\frac{1}{2}\left({ }_{a} D_{x}^{1-p} \phi \cdot \psi\right)_{x}, \quad \phi_{t}=\phi_{x x}+\frac{1}{2}{ }_{a} D_{x}^{p}\left[\left({ }_{a} D_{x}^{1-p} \phi\right)^{2}-\psi \psi_{x}\right]
$$

which are invariant in respect of supertransformations (12.1). In the general case, the system (12.3) is a system of two nonlinear nonlocal evolution equations, which becomes local when

$$
\begin{array}{ll}
p=0 & \left\{\begin{array}{l}
\psi_{t}=\psi_{x x}+\frac{1}{2}\left(\phi_{x} \psi\right)_{x}, \\
\phi_{t}=\phi_{x x}+\frac{1}{2}\left(\phi_{x}^{2}-\psi \psi_{x}\right),
\end{array}\right. \\
p=1 \quad\left\{\begin{array}{l}
\psi_{t}=\psi_{x x}+\frac{1}{2}(\phi \psi)_{x}, \\
\phi_{t}=\phi_{x x}+\frac{1}{2}\left(\phi^{2}-\psi \psi_{x}\right)_{x} .
\end{array}\right.
\end{array}
$$

The supersymmetric equation (12.2) and the corresponding system of equations (12.3) unite two fields of different nature, and only one of them is nonlocal.

Here, the main point is a general note related to the application of the nonlocal systems. Suppose the case when a dynamic system is characterized by two interacting fields, of them one, for instance, the "fermionic" field $\psi(x, t)$, is measured in the course of experiment, whereas the other, the "bosonic" field $\phi(x, t)$, is assessed only phenomenologically. Actually, such assessment in the class of local evolution equations may result in a qualitatively erroneous mathematical model of a dynamic system.

\section{Conclusions}

It is important to note that the influence of nonlocality can be arbitrarily great. Therefore, we do not describe nonlocality by an additional term in the Burgers equation.

Let us remind here that the classical Burgers equation belongs to a unique group of the three completely integrable second-order PDEs. I suggest that the FBENN also belongs to a unique group of the completely integrable nonlocal PDEs of the fractional order.

The fractional diffusion process is related to the non-Gauss statistics; this results in slow diffusion correlators $\left\langle(\Delta x)^{2}\right\rangle \propto D t^{\gamma}$ with $\gamma \neq 1$, and $D$ is a generalized diffusion coefficient of the dimension $L^{2} / T^{\gamma}$. In our case, the FBENN is related to the so-called Lévi statistics [39]; at the same time, the initial Burgers equation as well as the diffusion equation are related to the usual Gauss statistics.

From our point of view, the FBENN has at least two important advantages: 
i) the influence of nonlocality is not assumed to be insignificant;

ii) the relation of the FBENN to the usual diffusion equation allows a lot of analytical solutions of the FBENN.

Besides, despite the nonlocality in the proposed nonlinear and nonlocal FBENN,

- space-localized solutions are possible;

- nonlocal perturbations in a system described by the FBENN can interact;

- the Reynolds number is a universal dimensionless parameter for both the local and nonlocal Burgers equations;

- there are nonlocal analogies of both the momentum conservation law and kinetic energy dissipation.

In some fields of physics, we need the vectorial form of the Burgers equation, e.g., in astrophysics to describe the large-scale structure of the Universe [28, 29,43]. In such cases, the vectorial FBENN can be proposed

$$
\phi_{t}+\frac{1}{2}{ }_{a} D_{x}^{p}\left({ }_{a} D_{x}^{1-p} \phi\right)^{2}-\alpha \nabla(\nabla \phi)=0,
$$

where $\phi=\left(\phi^{1}, \ldots, \phi^{n}\right) \in \mathbb{R}^{n},{ }_{a} D_{x}^{p}$ is the fractional generalization of the gradient operator $\nabla$.

Note also that for $\alpha=0$ from FBENN (1.1) follows the fractional generalization of the Riemann equation, which also has numerous applications.

The proposed FBENN, because of its general character, allows a wide range of applications. Actually, we may try to introduce the nonlocal generalization in almost all fields where the BE is applied. These are the nonlocal effects in shock wave propagation in acoustics, the effective model of the process of nonlinear heat distribution in the environment in the presence of heat sources and sinks, the Kardar-Parisi-Zhang (KPZ) equation in the crystal growth phenomena in $(1+1)$-dimensions [23], the nonlinear dynamics of moving lines [22], formation of galaxies [29,38], the behavior of the magnetic flux line in superconductors [21], and spin glasses [13], as well as numerous examples of the application of the usual Burgers equation, presented in the abovementioned monographs $[17,41]$.

\section{A The normalized form of equations}

In applications, usually considered are both the non-normalized form of the diffusion equation with quadratic nonlinearity

$$
\phi_{t}+\alpha \phi_{x}^{2}-\beta \phi_{x x}=0
$$

and the Burgers equation

$$
\phi_{t}+\alpha \phi \phi_{x}-\beta \phi_{x x}=0
$$

in which the concrete sense of the coefficients $\alpha$ and $\beta$ depends on the content of a model under description. In the dimensional form, $\beta$ is normally related to the coefficient of diffusion, whereas in the dimensionless form it relates to the inverse Reynolds number $\mathrm{Re}^{-1}$.

Equations (A.1) and (A.2) in the non-normalized form, as well as the non-normalized equation FBENN (1.4)

$$
\phi_{t}+\alpha_{a} D_{x}^{p}\left({ }_{a} D_{x}^{1-p} \phi\right)^{2}-\beta \phi_{x x}=0,
$$


on performing the transformations

$$
\phi(x, t) \mapsto \frac{\beta}{\alpha} \phi(x, t), \quad t \mapsto \frac{1}{\beta} t,
$$

are reduced to the normalized form

$$
\phi_{t}+\phi_{x}^{2}+\phi_{x x}=0, \quad \phi_{t}+{ }_{a} D_{x}^{p}\left({ }_{a} D_{x}^{1-p} \phi\right)^{2}+\phi_{x x}=0, \quad \phi_{t}+\phi \phi_{x}+\phi_{x x}=0 .
$$

Note that in the case of the Burgers equation (for $p=1$ ) there exists a special transformation related to the changed scale of the independent variables without changing the function,

$$
x \mapsto \frac{\beta}{\alpha} x, \quad t \mapsto \frac{\beta}{\alpha^{2}} t,
$$

which allows also a reduction of the Burgers equation to a dimensionless form (5.1). Thus, now we may apply any form of the FBENN or its limit cases the BE or the NDE depending on our requirements.

\section{B The fractional calculus}

The left-side Riemann-Liouville fractional derivative of the order $0<\alpha<1$ is

$$
D_{a+}^{\alpha} f(t)=\frac{1}{\Gamma(1-\alpha)} \frac{d}{d t} \int_{a}^{t} \frac{f(\tau) d \tau}{(t-\tau)^{\alpha}} .
$$

The right-side fractional derivative is

$$
D_{b-}^{\alpha} f(t)=\frac{1}{\Gamma(1-\alpha)}\left(-\frac{d}{d t}\right) \int_{t}^{b} \frac{f(\tau) d \tau}{(\tau-t)^{\alpha}} .
$$

The left-side Riemann-Liouville fractional derivative of the arbitrary order $\alpha \in \mathbb{R}$ is

$$
D_{a+}^{\alpha} f(t)=\frac{1}{\Gamma(n-\alpha)}\left(\frac{d}{d t}\right)^{n} \int_{a}^{t} \frac{f(\tau) d \tau}{(t-\tau)^{1+\alpha-n}} .
$$

The right-side fractional derivative is

$$
D_{b-}^{\alpha} f(t)=\frac{1}{\Gamma(n-\alpha)}\left(-\frac{d}{d t}\right)^{n} \int_{t}^{b} \frac{f(\tau) d \tau}{(\tau-t)^{1+\alpha-n}}
$$

where $n=[\alpha]+1$.

The corresponding regularized fractional derivative of the function $f(x)$, or its Caputo fractional derivatives (in honour of M. Caputo [9]), are defined as follows

$$
{ }^{c} D_{a+}^{\alpha} f(t)=\frac{1}{\Gamma(1-\alpha)}\left[\frac{d}{d \tau} \int_{a}^{t} \frac{f(\tau) d \tau}{(t-\tau)^{\alpha}}-\frac{f(a)}{(t-a)^{\alpha}}\right],
$$

the right-side derivative being

$$
{ }^{c} D_{b-}^{\alpha} f(t)=\frac{1}{\Gamma(1-\alpha)}\left[\left(-\frac{d}{d \tau}\right) \int_{t}^{b} \frac{f(\tau) d \tau}{(\tau-t)^{\alpha}}-\frac{f(b)}{(b-t)^{\alpha}}\right] .
$$

The Caputo fractional derivative of the arbitrary order $\alpha \in \mathbb{R}$ is

$$
{ }^{c} D_{a+}^{\alpha} f(t)=\frac{1}{\Gamma(n-\alpha)}\left[\left(\frac{d}{d \tau}\right)^{n} \int_{a}^{t} \frac{f(\tau) d \tau}{(t-\tau)^{1+\alpha-n}}-\frac{f(a)}{(t-a)^{\alpha}}\right],
$$


and the right-side derivative is

$$
{ }^{c} D_{b-}^{\alpha} f(t)=\frac{1}{\Gamma(n-\alpha)}\left[\left(-\frac{d}{d \tau}\right)^{n} \int_{t}^{b} \frac{f(\tau) d \tau}{(\tau-t)^{1+\alpha-n}}-\frac{f(b)}{(b-t)^{\alpha}}\right] .
$$

If $f(x)$ is absolutely continuous on $[a, T]$ or corresponding $[T, b]$, then the left Caputo fractional derivative is

$$
{ }^{c} D_{a+}^{\alpha} f(t)=\frac{1}{\Gamma(n-\alpha)} \int_{a}^{t} \frac{d \tau}{(t-\tau)^{1+\alpha-n}}\left(\frac{d}{d \tau}\right)^{n} f(\tau),
$$

the right-side Caputo fractional derivative being

$$
{ }^{c} D_{b-}^{\alpha} f(t)=\frac{1}{\Gamma(n-\alpha)} \int_{t}^{b} \frac{d \tau}{(\tau-t)^{1+\alpha-n}}\left(-\frac{d}{d \tau}\right)^{n} f(\tau),
$$

where $\alpha$ represents the order of the derivative, $n-1<\alpha<n$.

The forms (B.3) and (B.4) are often used in physical literature. However, the forms (B.1) and (B.2) are more useful, since they may be applied to a wider class of functions.

The Caputo fractional derivative or integral in the Weyl sense is the corresponding Caputo operator for the absolutely continuous function $f(x)$ defined on the whole real axes $\mathbb{R}$.

Some properties of fractional derivatives and integrals are listed below

$$
\begin{aligned}
& D_{a+}^{-\alpha}=I_{a+}^{\alpha} f(t) \quad\left(D_{b-}^{-\alpha}=I_{b-}^{\alpha} f(t)\right), \quad \alpha>0, \\
& I_{a+}^{\alpha} f(t)=\frac{1}{\Gamma(\alpha)} \int_{a}^{t} \frac{f(\tau) d \tau}{(t-\tau)^{\alpha-1}}, \quad t>a, \\
& I_{b-}^{\alpha} f(t)=\frac{1}{\Gamma(\alpha)} \int_{t}^{b} \frac{f(\tau) d \tau}{(\tau-t)^{\alpha-1}}, \quad t<b . \\
& D_{a+}^{\alpha} f(t)=I_{a+}^{-\alpha} f(t), \\
& D_{a+}^{\alpha} D_{a+}^{\beta} f(t)=D_{a+}^{\beta} D_{a+}^{\alpha} f(t)=D_{a+}^{\alpha+\beta} f(t), \\
& I_{a+}^{\alpha} I_{a+}^{\beta} f(t)=I_{a+}^{\beta} I_{a+}^{\alpha} f(t)=I_{a+}^{\alpha+\beta} f(t), \\
& f(t)=\sum_{j=0}^{n-1} \frac{D_{a+}^{\alpha+j} f(0)}{\Gamma(1+\alpha+j)} t^{\alpha+j}+R_{n}(t), \quad n=[\operatorname{Re} \alpha]+1,
\end{aligned}
$$

where $R_{n}(t)=I_{a+}^{\alpha+j} D_{a+}^{\alpha+j} f(t)$.

The derivatives of some functions are:

$$
{ }_{-\infty} D_{t+}^{\alpha} \sin \lambda t=\lambda^{\alpha} \sin \left(\lambda t+\frac{\pi \alpha}{2}\right), \quad{ }_{-\infty} D_{t+}^{\alpha} \cos \lambda t=\lambda^{\alpha} \cos \left(\lambda t+\frac{\pi \alpha}{2}\right),
$$

where $\lambda>0, \alpha>-1$; when $\alpha \leq-1$, we have to use the property (B.5);

$$
{ }_{-\infty} D_{t+}^{\alpha} \mathrm{e}^{\lambda t+\mu}=\lambda^{\alpha} \mathrm{e}^{\lambda t+\mu}, \quad \operatorname{Re} \lambda>0 .
$$

The Riesz fractional derivative and integral of order $\alpha$ are defined by the Fourier transformation

$$
\mathrm{D}_{x}^{\alpha} f(x)=\mathcal{F}^{-1}\left(|k|^{\alpha}(\mathcal{F} f)(k)\right), \quad \mathrm{I}_{x}^{\alpha} f(x)=\mathcal{F}^{-1}\left(|k|^{-\alpha}(\mathcal{F} f)(k)\right) .
$$

The Riesz fractional integral could be presented as a convolution

$$
\mathrm{I}_{x}^{\alpha} f(x)=\int_{\mathbb{R}^{n}} K_{\alpha}(x-\xi) f(\xi) d \xi, \quad \alpha>0,
$$


where $K_{\alpha}(x)$ is the Riesz kernel

$$
K_{\alpha}(x)= \begin{cases}\gamma_{n}^{-1}(\alpha)|x|^{\alpha-n}, & \alpha \neq n+2 k, \\ -\gamma_{n}^{-1}(\alpha)|x|^{\alpha-n} \ln |x|, & \alpha=n+2 k,\end{cases}
$$

where $k \in \mathbb{N}$ and coefficients $\gamma_{n}^{-1}(\alpha)$ are

$$
\gamma_{n}^{-1}(\alpha)= \begin{cases}2^{\alpha} \pi^{n / 2} \Gamma\left(\frac{\alpha}{2}\right) / \Gamma\left(\frac{n-\alpha}{2}\right), & \alpha \neq n+2 k, \\ (-1)^{(n-\alpha) / 2} 2^{\alpha-1} \pi^{n / 2} \Gamma\left(\frac{\alpha}{2}\right) \Gamma\left(1+\frac{\alpha-n}{2}\right), & \alpha=n+2 k .\end{cases}
$$

Note that the correlation of the Riesz and Caputo fractional derivatives in the Weyl sense

$$
\mathrm{D}_{x}^{\alpha} f(x)=\frac{1}{2 \cos (\alpha \pi / 2)}\left({ }^{c} D_{+}^{\alpha} f(x)+{ }^{c} D_{-}^{\alpha} f(x)\right) .
$$

Two special functions of those often used in fractional calculus are as follows: the Mittag-Leffler function

$$
E_{\alpha, \beta}(z)=\sum_{n=0}^{\infty} \frac{z^{n}}{\Gamma(\alpha n+\beta)}
$$

the generalized exponential function

$$
E_{\alpha}^{z}=\sum_{n=0}^{\infty} \frac{z^{n+\alpha}}{\Gamma(1+\alpha+n)}
$$

The basic aspects of fractional calculus and its applications could be found in $[3,10,16,19$, $25,27,28,30,33,37,42]$.

\section{Supersymmetry}

Let us consider an important example. Two large infinite classes of groups, $\operatorname{Osp}(N \mid M)$ and $\mathrm{SU}(N \mid M)$, are used in applications. The orthogonal group $\mathrm{O}(N)$ preserves the invariant $x_{i} x^{i}$, and the group $\operatorname{Sp}(M)$ retains the invariant $\theta_{m} C_{m n} \theta^{n}$, where the $C_{m n}$ matrices are real antisymmetric matrices and $\theta_{i}$ are Grassmann-valued. The orthosymplectic group is now defined as the group that preserves the sum

$$
\operatorname{Osp}(N \mid M): x_{i} x^{i}+\theta_{m} C_{m n} \theta^{n}=\operatorname{inv} .
$$

Note that the orthosymplectic group contains the product

$$
\operatorname{Osp}(N \mid M)=\left(\begin{array}{cc}
\mathrm{O}(N) & A \\
B & \mathrm{Sp}(M)
\end{array}\right)
$$

Let us write the generators of $\operatorname{Osp}(1 \mid 4)$ as $M_{A}=\left(p_{\mu}, M_{\mu, \nu}, Q_{\alpha}\right)$ which have the commutation relations

$$
\left[M_{A}, M_{B}\right]_{ \pm}=f_{A B}^{C} M_{C}
$$

In the explicit form, the commutators involving the supersymmetry generator are

$$
\left\{Q_{\alpha}, Q_{\beta}\right\}=2\left(\gamma^{\mu} C\right)_{\alpha \beta} P_{\mu}, \quad\left[Q_{\alpha}, P_{\mu}\right]=0, \quad\left[Q_{\alpha}, M_{\mu, \nu}\right]=\left(\sigma_{\mu, \nu}\right)_{\alpha}^{\beta} Q_{\beta} .
$$


Let us define superspace as the space created by the pair $x_{\mu} \theta_{\alpha}$, where $\theta_{\alpha}$ is a Grassmann number. Let us define the supersymmetry generator as

$$
Q_{\alpha}=\frac{\partial}{\partial \bar{\theta}^{\alpha}}-i\left(\gamma^{\mu} \theta\right)_{\alpha} \partial_{\mu}
$$

The anticommutator between two such generators yields a displacement

$$
\left\{Q_{\alpha}, Q_{\beta}\right\}=-2\left(\gamma^{\mu} C\right)_{\alpha \beta} i \partial_{\mu} .
$$

Note that $\bar{\varepsilon} Q$ makes the following transformations of the superspace

$$
x_{\mu} \rightarrow x_{\mu}-i \bar{\varepsilon} \gamma_{\mu} \theta, \quad \theta_{\alpha} \rightarrow \theta_{\alpha}+\varepsilon_{\alpha} .
$$

We can construct the operator

$$
D_{\alpha}=\frac{\partial}{\partial \bar{\theta}^{\alpha}}+i\left(\gamma^{\mu} \partial_{\mu} \theta\right)_{\alpha}
$$

This operator $D_{\alpha}$ anticommutes with the supersymmetry generator, $\left\{Q_{\alpha}, D_{\beta}\right\}=0$. This relation is very important because it allows us to place restrictions on the representations of supersymmetry without destroying the symmetry. This permits us to extract the irreducible representations from the reducible ones.

\section{Acknowledgements}

The author would like to express his gratitude to Professors B.A. Dubrovin, M. Pavlov and L. Alaniya for the invitation and kind hospitality during the Conference "Geometrical Methods in Mathematical Physics" (Moscow State University, December 12-17, 2011).

\section{References}

[1] Abraham-Shrauner B., Guo A., Hidden and nonlocal symmetries of nonlinear differential equations, in Modern Group Analysis: Advanced Analytical and Computational Methods in Mathematical Physics (Acireale, 1992), Editors N.H. Ibragimov, M. Torrisi, A. Valenti, Kluwer Acad. Publ., Dordrecht, 1993, 1-5.

[2] Agarwal R.P., O'Regan D. (Editors), Integral and integrodifferential equations. Theory, methods and applications, Series in Mathematical Analysis and Applications, Vol. 2, Gordon and Breach Science Publishers, Amsterdam, 2000.

[3] Bardou F., Bouchaud J.-P., Aspect A., Cohen-Tannoudji C., Lévy statistics and laser cooling. How rare events bring atoms to rest, Cambridge University Press, Cambridge, 2002.

[4] Bateman H., Some recent researches in the motion of fluids, Monthly Weather Rev. 43 (1915), $163-167$.

[5] Bertotti G., Hysteresis in magnetism: for physicists, materials scientists, and engineers, Academic Press, New York, 1998.

[6] Biler P., Funaki T., Woyczynski W.A., Fractal Burgers equations, J. Differential Equations 148 (1998), 9-46.

[7] Biler P., Karch G., Woyczyński W.A., Critical nonlinearity exponent and self-similar asymptotics for Lévy conservation laws, Ann. Inst. H. Poincaré Anal. Non Linéaire 18 (2001), 613-637.

[8] Burgers J.M., The nonlinear diffusion equation. Asymptotic solutions and statistical problems, D. Reidel Publishing Company, Dordrecht, 1974.

[9] Caputo M., Linear model of dissipation whose $Q$ is almost frequency independent. II, Geophys. J. R. Astronom. Soc. 13 (1967), 529-539.

[10] Carpinteri A., Mainardi F. (Editors), Fractals and fractional calculus in continuum mechanics, CISM Courses and Lectures, Vol. 378, Springer-Verlag, Vienna, 1997. 
[11] Clarkson P.A., Mansfield E.L., Symmetries of the nonlinear heat equation, in Modern Group Analysis: Advanced Analytical and Computational Methods in Mathematical Physics (Acireale, 1992), Editors N.H. Ibragimov, M. Torrisi, A. Valenti, Kluwer Acad. Publ., Dordrecht, 1993, 155-171.

[12] Cole J.D., On a quasi-linear parabolic equation occurring in aerodynamics, Quart. Appl. Math. 9 (1951), 225-236.

[13] Fisher D.S., Huse D.A., Directed paths in a random potential, Phys. Rev. B 43 (1991), 10728-10742.

[14] Fokas A.S., Zakharov V.E. (Editors), Important developments in soliton theory, Springer Series in Nonlinear Dynamics, Springer-Verlag, Berlin, 1993.

[15] Galaktionov V.A., Posashkov S.A., New exact solutions of parabolic equations with quadratic nonlinearities, U.S.S.R. Comput. Math. and Math. Phys. 29 (1989), 112-119.

[16] Gerasimov A.N., A generalization of linear laws of deformation and its application to problems of internal friction, Prikl. Mat. Meh. 12 (1948), 251-260.

[17] Gurbatov S.N., Malakhov A.N., Saichev A.I., Nonlinear random waves and turbulence in nondispersive media: waves, rays, particles, Nonlinear Science: Theory and Applications, Manchester University Press, Manchester, 1991.

[18] Hernández Heredero R., Levi D., Winternitz P., Symmetry preserving discretization of the Burgers equation, in SIDE III - Symmetries and Integrability of Difference Equations (Sabaudia, 1998), CRM Proc. Lecture Notes, Vol. 25, Amer. Math. Soc., Providence, RI, 2000, 197-208.

[19] Hilfer R. (Editor), Applications of fractional calculus in physics, World Scientific Publishing Co. Inc., River Edge, NJ, 2000.

[20] Hopf E., The partial differential equation $u_{t}+u u_{x}=\mu u_{x x}$, Comm. Pure Appl. Math. 3 (1950), 201-230.

[21] Hwa T., Nonequilibrium dynamics of driven line liquids, Phys. Rev. Lett. 69 (1992), 1552-1555, condmat/9206008.

[22] Hwa T., Kardar M., Avalanches, hydrodynamics, and discharge events in models of sandpiles, Phys. Rev. A 45 (1992), 7002-7023.

[23] Kardar M., Parisi G., Zhang Y.-C., Dynamic scaling of growing interfaces, Phys. Rev. Lett. 56 (1986), 889-892.

[24] Kilbas A.A., Generalized fractional differential and difference equations, in Fractional Integral Equations, Proceedinds AMAD-2003, Belarusian State University, Minsk, 2003, 5-13.

[25] Klafter J., Shlesinger M.F., Zumofen G., Beyond Brownian motion, Phys. Today 49 (1996), no. 2, 33-39.

[26] Lakes R.S., Viscoelastic solids, CRC Mechanical Engineering Series, CRC Press, New York, 1998.

[27] Miller K.S., Ross B., An introduction to the fractional calculus and fractional differential equations, $A$ WileyInterscience Publication, John Wiley \& Sons Inc., New York, 1993.

[28] Miškinis P., Nonlinear and nonlocal integrable models, Technika, Vilnius, 2003.

[29] Miškinis P., The nonlinear heterogeneous diffusion equation and distribution of matter in the early Universe, Astrophys. J. Lett. 543 (2000), L95-L98.

[30] Nigmatullin R.R., To the theoretical explanation of the "universal response", Phys. Stat. Sol. B 123 (1984), 739-745.

[31] Novikov S., Manakov S.V., Pitaevskiǔ L.P., Zakharov V.E., Theory of solitons. The inverse scattering method, Contemporary Soviet Mathematics, Plenum, New York, 1984.

[32] Oldham K.B., Spanier J., The fractional calculus. Theory and applications of differentiation and integration to arbitrary order, Mathematics in Science and Engineering, Vol. 111, Academic Press, New York - London, 1974 .

[33] Podlubny I., Fractional differential equations. An introduction to fractional derivatives, fractional differential equations, to methods of their solution and some of their applications, Mathematics in Science and Engineering, Vol. 198, Academic Press, San Diego, CA, 1999.

[34] Polyanin A.D., Manzhirov A.V., Handbook of integral equations, CRC Press, Boca Raton, FL, 1998.

[35] Riemann B., Ueber die Fortpflanzung ebener Luftwellen von endlicher Schwingungsweite, Abh. Königl. Ges. Wiss. Göttingen 8 (1860), 43-65. 
[36] Sakhnovich L.A., Integral equations with difference kernels on finite intervals, Operator Theory: Advances and Applications, Vol. 84, Birkhäuser Verlag, Basel, 1996.

[37] Samko S.G., Kilbas A.A., Marichev O.I., Fractional integrals and derivatives. Theory and applications, Gordon and Breach Science Publishers, Yverdon, 1993.

[38] Shandarin S.F., Zel'dovich Ya.B., The large-scale structure of the universe: turbulence, intermittency, structures in a self-gravitating medium, Rev. Modern Phys. 61 (1989), 185-220.

[39] Shlesinger M.F., Zaslavsky G.M., Frisch U. (Editors), Lévy flights and related topics in physics, Lecture Notes in Physics, Vol. 450, Springer-Verlag, Berlin, 1995.

[40] Volterra V., Lecons sur la théorie mathématique de la lutte pour la vie, Gauthier-Villars, Paris, 1931.

[41] Woyczyński W.A., Burgers-KPZ turbulence. Göttingen lectures, Lecture Notes in Mathematics, Vol. 1700, Springer-Verlag, Berlin, 1998.

[42] Zaslavsky G.M., Chaos, fractional kinetics, and anomalous transport, Phys. Rep. 371 (2002), 461-580.

[43] Zel'dovich Ya.B., Gravitational instability: an approximate theory for large density perturbations, Astronom. Astrophys. 5 (1970), 84-95. 